\title{
Morphologic characteristics and clinical significance of the macular-sparing area in patients with retinitis pigmentosa as revealed by multicolor imaging
}

\author{
GUODONG LIU*, QING DU*, KHUSBU KEYAL and FANG WANG \\ Department of Ophthalmology, Shanghai Tenth People's Hospital Affiliated to Tongji University School \\ of Medicine, Shanghai 200072, P.R. China
}

Received May 13, 2017; Accepted August 14, 2017

DOI: $10.3892 /$ etm.2017.5227

\begin{abstract}
Retinitis pigmentosa (RP) is an inherited retinal disease caused by the degeneration of photoreceptors and retinal pigment epithelium cells within the eye. The present study aimed to evaluate the effectiveness of multicolor imaging as a novel technique for the depiction of morphological features in the macular area of patients with RP. Additionally, the correlation between the size of the macular-sparing area and the best corrected visual acuity (BCVA), central visual field function and subfoveal choroidal thickness (SFCT) was analyzed. A total of 25 individuals with RP ( $\mathrm{n}=50$ eyes) and 35 healthy individuals ( $\mathrm{n}=70$ eyes) were enrolled in the current study. Images of the macular area were captured using multicolor imaging and traditional fundus photography, and their ability to depict retinal features was compared. The size of the macular-sparing area in patients with RP was measured using built-in measurement software programs. The correlations between the macular-sparing area and the BCVA, visual field and SFCT were analyzed using a Spearman's rank correlation test. The results demonstrated that multicolor imaging revealed clinical features of the macular area in greater detail than traditional fundus photography irrespective of whether the patient also had complications, including cataracts and epiretinal membranes. Multicolor imaging clearly defined the borders of the macular-sparing area corresponding to the relatively intact outer retinal structures on optical coherence tomography images, particularly the status of the ellipsoid zone and external limiting membrane. There was a significant
\end{abstract}

Correspondence to: Dr Fang Wang, Department of Ophthalmology, Shanghai Tenth People's Hospital Affiliated to Tongji University School of Medicine, 301 Middle Yan Chang Road, Shanghai 200072, P.R. China

E-mail: 18917683335@163.com

${ }^{*}$ Contributed equally

Key words: multicolor imaging, optical coherence tomography, retinitis pigmentosa, macular-sparing area positive correlation between the macular-sparing area and BCVA $(r=-0.631 ; \mathrm{P}<0.001)$, and the visual field in terms of $\mathrm{MD}(\mathrm{r}=0.402 ; \mathrm{P}<0.05)$ and PSD $(\mathrm{r}=0.595 ; \mathrm{P}<0.001)$, however, there was not a statistically significant correlation between the macular-sparing area and SFCT. The present study demonstrated that multicolor imaging is capable of detecting macular changes and complications in patients with RP. Multicolor imaging may be particularly useful in assessing the detailed characteristics of the macular-sparing area, as this appears to be associated with visual function.

\section{Introduction}

Retinitis pigmentosa (RP) is an inherited retinal disease caused by the degeneration of photoreceptors and retinal pigment epithelium (RPE) within the eye (1). RP is a major cause of acquired blindness in adults worldwide, occurring in 1 out of every 4,000 individuals (2). RP is characterized by nyctalopia, progressive visual field contraction and, ultimately, central visual field loss (3). A clinical diagnosis of RP is largely based on characteristic fundus changes, decreased electroretinography (ERG) responses and optical coherence tomography (OCT) examinations. Previous histological studies have determined that the death of RPE cells and photoreceptors is the common progression for all patients with RP. However, while certain patients with RP lose their vision at a young age, others maintain central vision for their entire lives without treatment (3). The RP patients with central vision are able to maintain a relatively normal macular area for an extended period of time, which is defined as the 'macular-sparing area' throughout the present study. Visual field function is associated with the type of macular lesion the patient has (4) and may be assessed by the observation of preserved outer retinal lines (5).

With the introduction of spectral domain (SD)-OCT, it has been possible to obtain structural information on retinal anatomical abnormalities in patients with RP (6). Previous studies have demonstrated that three highly reflective lines, including the external limiting membrane (ELM), the ellipsoid zone (EZ) and the interdigitation zone in the outer retina are important predictors of visual acuity $(7,8)$, which is highly correlated with visual field function (9). However, patients with 
RP often have complications, including cataracts, epiretinal membranes (ERM) and macular edema $(10,11)$, which makes it difficult to acquire useful information from traditional fundus images or ophthalmoscopy.

Heidelberg Engineering GmbH (Heidelberg, Germany) introduced a novel combined instrument, the Spectralis SD-OCT, which enables the recording of confocal scanning laser ophthalmoscope (cSLO) and SD-OCT images simultaneously (12). Multicolor imaging uses a cSLO with three laser wavelengths to acquire reflective images (blue reflectance, $488 \mathrm{~nm}$; green reflectance, $515 \mathrm{~nm}$; infrared (IR) reflectance, $820 \mathrm{~nm}$ ). The Spectralis SD-OCT is able to obtain detailed structural information and localize pathologic abnormalities more precisely than previously available instruments. The present study evaluated the effectiveness of the Spectralis SD-OCT in defining the macular-sparing area by multicolor imaging compared with traditional fundus photography. The potential correlations between the macular-sparing area and the best corrected visual acuity (BCVA), the visual field and subfoveal choroidal thickness (SFCT) were also analyzed.

\section{Materials and methods}

Inclusion of patients. Between January 2015 and June 2017, 25 patients with RP (RP group; $n=50$ eyes) and 35 healthy individuals (control group; $n=70$ eyes) were enrolled in the present study at Shanghai Tenth People's Hospital Affiliated to Tongji University School of Medicine (Shanghai, China). All participants in the present study underwent a complete ophthalmic examination, including a BCVA test, slit lamp biomicroscopy, an intraocular pressure test, a dilated fundus examination and SD-OCT. Patients with RP were further tested with full-field ERG, and central visual field and muticolor imaging.

Patients with RP were diagnosed based on the presence of nyctalopia, characteristic funduscopic appearance, constricted visual field and a reduction in full-field ERG. Individuals with significant media opacities and other intraocular diseases were excluded from the current study. BCVA is expressed as the logarithm of the minimum angle of resolution ( $\log M A R)$. For calculations, the BCVA of finger counts and hand movement were designated as 1.6 and 2.0, as previously described (13). The control group participants had a visual acuity of $\geq 20 / 20$, ametropic value of $<-3.00 \mathrm{D}$ and no ocular or systemic diseases.

Written informed consent was obtained from all participants. Procedures followed the tenets of the Declaration of Helsinki and the present study was approved by the Institutional Review Board of Shanghai Tenth People's Hospital.

Multicolor imaging. All patients with RP underwent multicolor imaging using the Spectralis SD-OCT (version 6.0.9) within the central 30 degrees of the visual field. Multicolor imaging uses multiple colored lasers to selectively capture information on different retinal structures. The blue laser reflectance produces detailed views of the retinal nerve fiber layers and epiretinal abnormalities. The green laser reflectance provides information on the retinal vasculature and intra-retinal structures, while the IR laser penetrates into the deepest retinal layers, providing images of the choroid, RPE cells and photoreceptors (14). Multicolor images are composed of the reflective images acquired by the three different colored lasers. SD-OCT scanning combined with multicolor imaging was performed simultaneously by the Spectralis SD-OCT.

Macular-sparing area measurement. The macular-sparing areas were traced independently by two physicians. The built-in measurement software programs within the Spectralis SD-OCT subsequently converted the pixels selected in the IR images to $\mathrm{mm}^{2}$. The average data from the results was used for analysis.

SFCT measurement. Enhanced depth imaging scans were used to assess the SFCT. Single line scans composed of 100 averaged images were captured to achieve a high quality image. A horizontal scan across the fovea was selected for the RP and control group, since this is a precise method for measuring subfoveal choridal thickness (15). Two experienced OCT readers measured the SFCT from the inner border of the sclera to the outer border of the RPE. Detailed information, including serial number, name, sex and date of birth, was recorded to ensure that individual participants could be identified following data collection.

Visual field. Static automated perimetry (central 30-2 threshold program with spot size III; Humphrey 750i; Carl Zeiss Meditec, Dublin, CA, USA) was performed on patients with RP to determine their visual field function. The visual field test was performed once per eye prior to the baseline test to minimize practice effects. To decrease the duration of the examination a Swedish interactive threshold algorithm fast strategy was used. Mean deviation (MD) and pattern standard deviation (PSD) data were obtained to assess the function of the visual field. MD represents the average deviation of sensitivity from age-corrected threshold values, while PSD represents the contiguous irregularities and the increased value with more irregularities (16).

Statistical analysis. All data were analyzed using SPSS software (version 20.0; IBM Corp., Armonk, NY, USA). An independent samples t-test was used for comparison of SFCT between the patients with RP and the controls. The associations between the macular-sparing area and BCVA, visual field and SFCT were measured using a Spearman's rank correlation test. $\mathrm{P}<0.05$ was considered to indicate a statistically significant difference.

\section{Results}

Participant inclusion within the study. In total, 25 patients with RP (50 eyes) underwent ophthalmic examinations. There were 4 eyes excluded due to nystagmus and 2 eyes excluded due to severe cataracts. Overall, 44 eyes of 24 patients with RP (14 male and 10 female; age, 26-80 years; mean age, $51.11 \pm 13.15$ years) were analyzed in the present study. A total of 70 eyes from 35 healthy participants (20 male and 15 female; age, $25-71$ years; mean age, $57.51 \pm 10.19$ years) were examined as the control group. No significant differences were identified in age and gender between the RP group and the control group. All patients in the RP group had concentric constrictions of the visual field. The mean MD and PSD were $-28.40 \pm 4.01 \mathrm{~dB}$ and $6.32 \pm 3.62 \mathrm{~dB}$, respectively. 

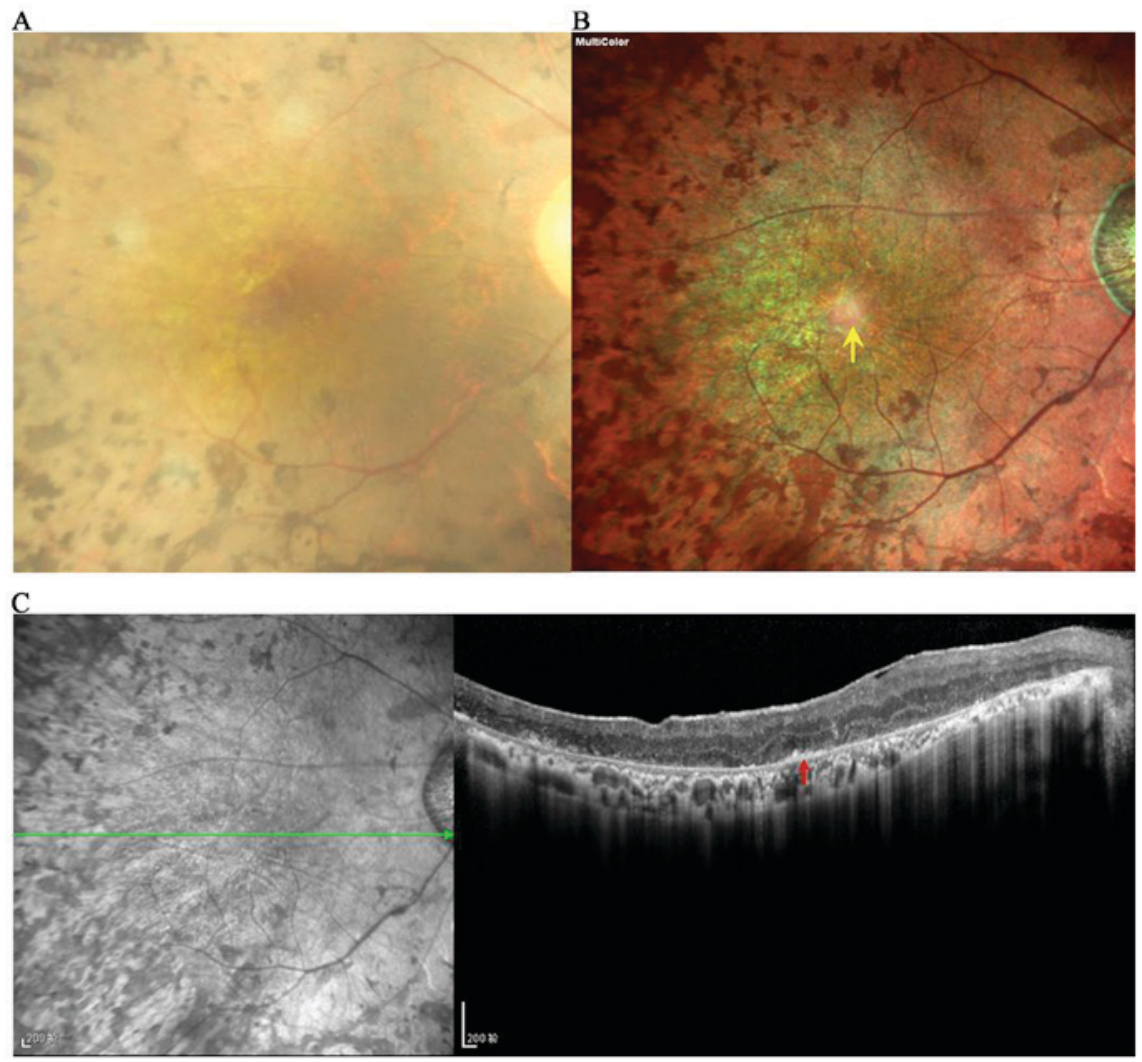

Figure 1. Comparison of fundus photography and multicolor imaging in the evaluation of the macular area of a patient with RP and ERM. An image of the macular area of a patient with RP and ERM captured using (A) traditional fundus photography and (B) multicolor imaging. The radiating perifoveal retinal folds, tractional retinal area were clearly visible on the multicolor cSLO image, and the yellow arrow indicates the sparing macular fovea. (C) The green line shows a horizontal optical coherence tomography scan corresponding with the multicolor cSLO image, revealing the resolution of ERM with a thickened macula. Red arrow indicates disrupted retinal pigment epithelium layer and outer retinal lines in the macular foveal. RP, retinitis pigmentosa; cSLO, confocal scanning laser ophthalmoscope; ERM, epiretinal membrane.

Use of multicolor imaging in the evaluation of the macular area of a patient with RP with an ERM. Traditional fundus photography (Fig. 1A) was used to identify the abnormal macular area, however, the retinal aberrations were difficult to detect due to the presence of moderate cataracts. Conversely, the multicolor cSLO image clearly revealed the presence of an ERM and macular aberrations (Fig. 1B). The multicolor cSLO image exposed a green macular area with regular elliptical shape boundaries, indicating the thickening of the macular retina. Spicule-shaped pigment deposits were also identified surrounding the macular area. The multicolor image demonstrates the radiating retinal folds in the ERM and a moderately healthy macular fovea within the area. A horizontal OCT B-scan corresponding to the multicolor image is presented in Fig. 1C, which revealed the ERM, an irregular RNFL layer, thickening of the macular fovea, a preserved RPE layer and outer retinal lines.

Multicolor imaging for the evaluation of the macular-sparing area of a patient with RP with a mild cataract. An individual from the RP group with a regular macular area and a mild cataract was selected to evaluate the structural changes of RP using multicolor imaging. In the image captured using fundus photography it was difficult to distinguish the exact border of the macular-sparing area due to the cataract and tissue overlay (Fig. 2A). By contrast, multicolor imaging is able to acquire structural information from different layers, and the oblong margins of the area are well defined on the multicolor image (Fig. 2B). The green line on the IR image (Fig. 2C) reveals the location of the horizontal cross-sectional SD-OCT image corresponding to the adjacent image. The red arrowheads indicate the horizontal boundaries of the normal reflective area, while the borders of the hyporeflective ring are shown between the yellow arrowheads on the IR image. The same anatomical locations of the normal reflective area indicate the region of the EZ band, while the hyporeflective ring is characterized by the disrupted ELM line on the SD-OCT image. While the ELM length was significantly longer than EZ length within the area, the ELM and EZ lines were absent outside of the hyporeflective ring.

Multimodal imaging for the evaluation of the macular-sparing area of a patient with RP. When compared with the fundus photography image in Fig. 3A, the margins of the macular-sparing area were well visualized on the multicolor image and disrupted RPE changes with leopard-spot pigment deposits can be clearly observed in the periphery of the retina (Fig. 3B). The multicolor image reveals a normal reflective area surrounded by a hyperreflective ring. The horizontal OCT exhibits the continuous outer retinal lines (EZ and ELM) within the normal reflective area, while the hyperreflective ring corresponds to a thickening ELM area, and the hyporeflective area coincides with the disrupted ELM line and invisible EZ line (Fig 3C). Multicolor imaging was able to provide high 

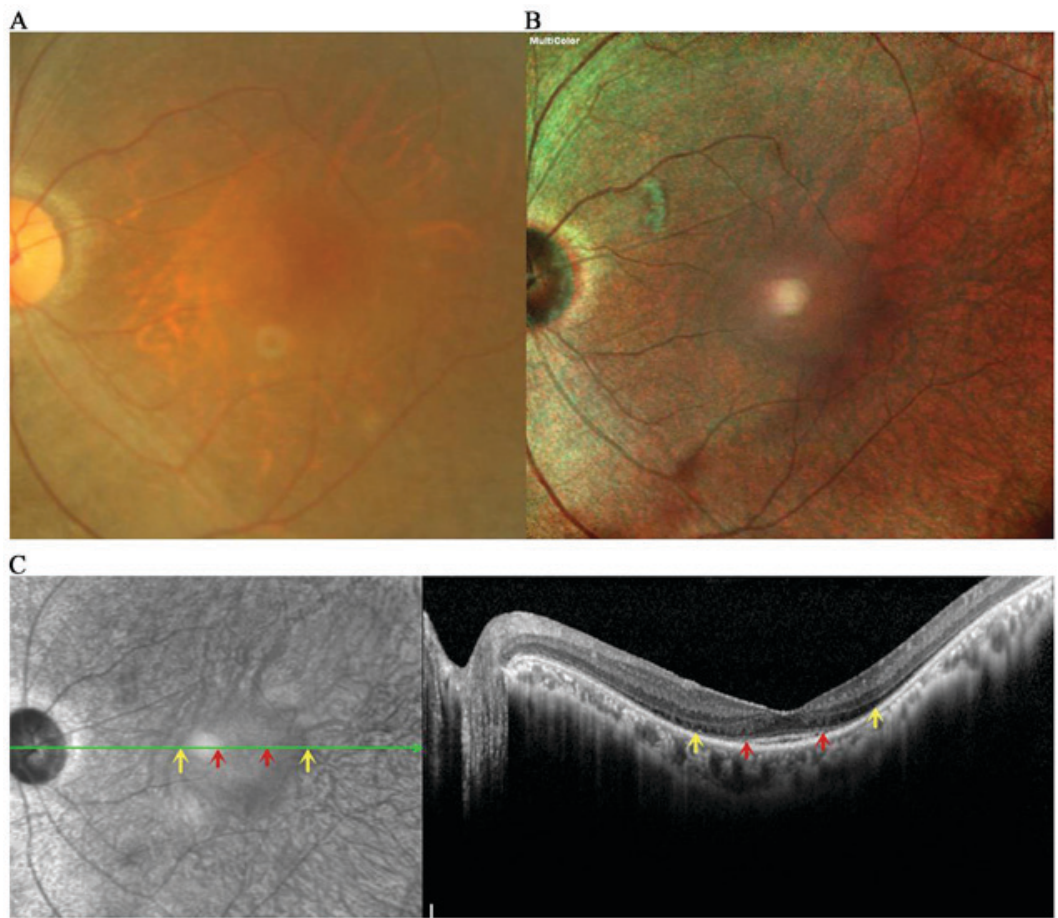

Figure 2. Comparison of fundus photography and multicolor imaging in the depiction of retinal features in a patient with RP and cataracts. An image of the retina of a patient with RP and a macular-sparing area captured using (A) traditional fundus photography and (B) multicolor imaging. The margins of the macular-sparing area concurrent with a mild cataract were relatively indistinct on traditional fundus photography, while the oblong margins of the macular-sparing area were clearly defined on the multicolor cSLO image. (C) The green line indicates a spectral-domain optical coherence tomography corresponding with the multicolor cSLO image. The red arrowheads show the horizontal boundaries of the normal outer retina layers, while yellow arrowheads indicate the borders of the external limiting membrane, which are at same anatomical location in the multicolor cSLO image. RP, retinal pigmentosa; cSLO, confocal scanning laser ophthalmoscope.
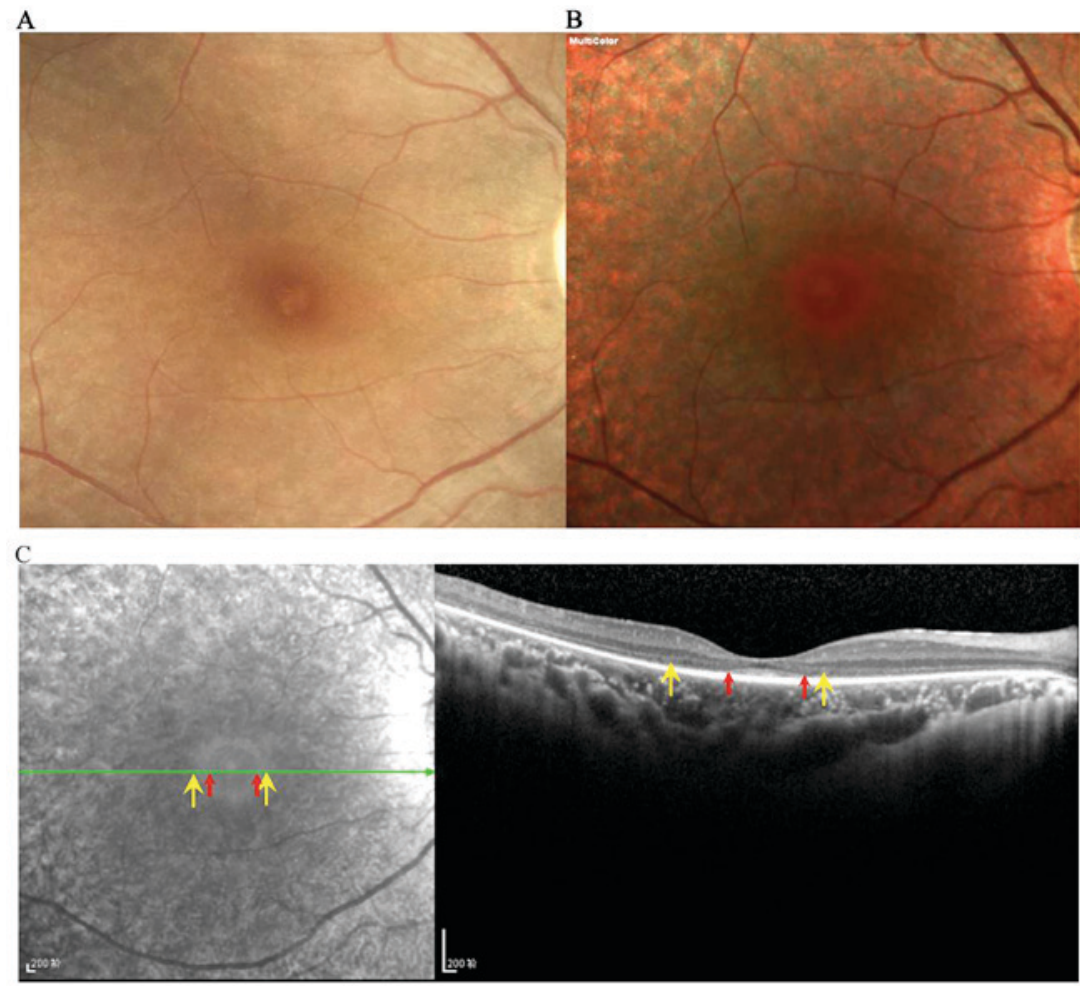

Figure 3. Comparison of fundus photography and multicolor imaging in the depiction of the macular-sparing area of a patient with RP. An image of the macular-sparing area of a patient with RP captured using (A) traditional fundus photography and (B) multicolor imaging. The borders of the macular-sparing area were not definable on the fundus photograph, while the multicolor image depicted the area clearly. (C) The green line shows a horizontal optical coherence tomography scan, and red arrows indicate continuous outer retinal lines (EZ and ELM) within the normal reflective area, while yellow arrows demonstrate hyper-reflective ring corresponding to thickened ELM line and the hypo-reflective area coincided with the disrupted ELM line and absent EZ line can be seen outside the yellow arrows. RP, retinal pigmentosa; EZ, ellipsoid zone; ELM, external limiting membrane. 

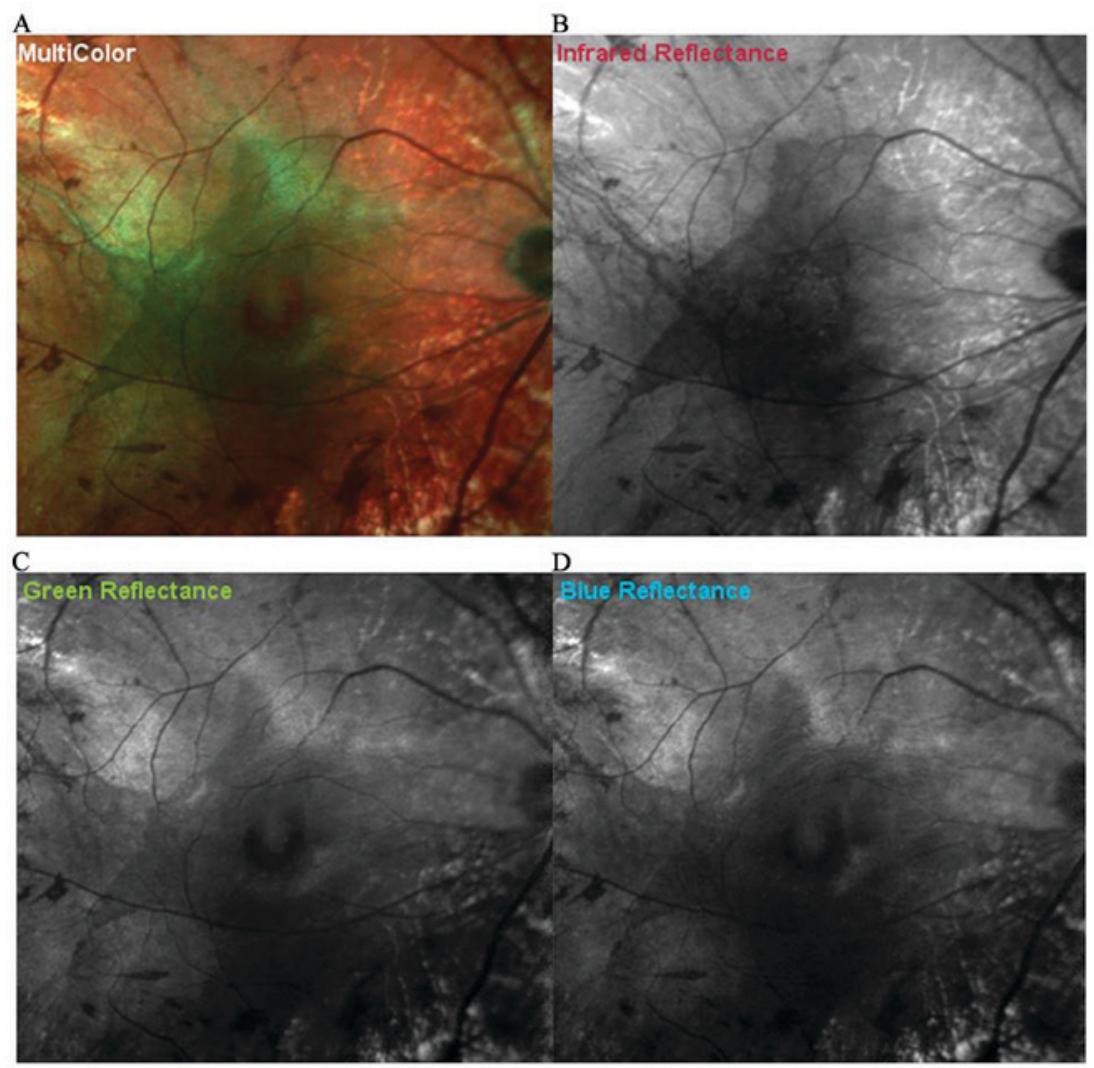

Figure 4. Breakdown of multicolor imaging with differently colored lasers. The baseline characteristics of the macular area are depicted using (A) multicolor imaging. (B) The irregular margins of the outer retinal alterations were best appreciated on the infrared reflectance, and were moderately visualized on the (C) green and (D) blue reflectance images.

resolution images of the macular-sparing area and visualize more details relating to retinal alterations, particularly the outer retinal changes, compared with fundus photography.

Multicolor imaging for the evaluation of macular features in a patient with RP with irregular margins. Multimodal images depict features of the fundus. The macular-sparing area is characterized by a green area on the multicolor image (Fig. 4A), which indicates a thickening retina or relatively preserved RPE layer. The IR reflectance image exhibited a high intergraded agreement of hyporeflective area with the multicolor image (Fig 4B). The image also revealed abrupt margins in the nasal and superonasal quadrants, and increased choroidal signals because of the disruption and loss of photoreceptors and RPE cells. The outer retinal features are moderately visualized on the green and blue reflectance images (Fig. 4C and D). The macular-sparing area was better outlined on the infrared reflectance image. Within the macular fovea, the heterogeneous reflectance images indicated the irregular outer retinal structures. Surrounding the macular area, bone spicule-shaped pigment deposits could be observed on all reflectance images (Fig. 4).

Macular-sparing area measurements. The macular-sparing area was traced independently by two observers on the IR images (Fig. 5) using built-in measurement software. The size of the area was calculated by an automatic conversion of pixels to $\mathrm{mm}^{2}$. The mean size of the area was $18.80 \pm 17.72 \mathrm{~mm}^{2}$ in the RP group (data not shown). The size in male patients was $15.99 \pm 16.55 \mathrm{~mm}^{2}$, while in female patients it was

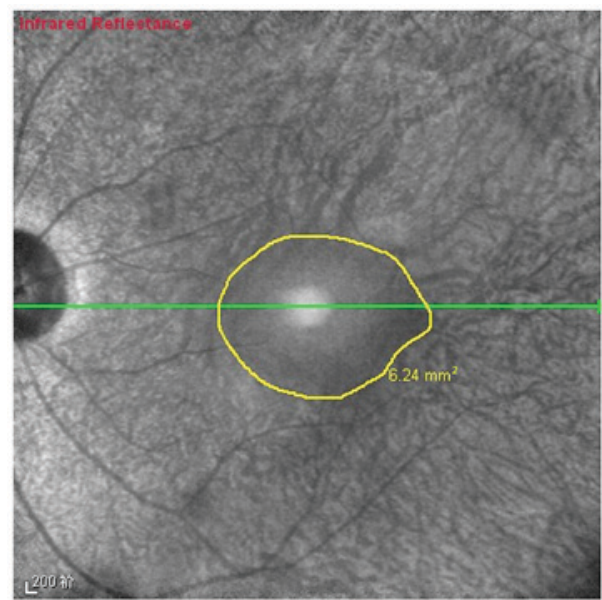

Figure 5. Measurements of the macular-sparing area. The macular-sparing area was traced manually by two independent observers and them measured by the built-in measurement software programs. The size of the macular-sparing area was calculated by automatic conversion of pixels into $\mathrm{mm}^{2}$. The average data was used for analysis. The yellow circle shows the preserved macular area in a left eye of a patient with retinal pigmentosa, and the size is $6.24 \mathrm{~mm}^{2}$.

$22.32 \pm 19.03 \mathrm{~mm}^{2}$ (data not shown). These results demonstrate that female patients exhibited a larger macular area, but that the difference was not significant (data not shown).

SFCT measurements. SFCT was measured from the inner border of the sclera to the outer border of the RPE vertically. 

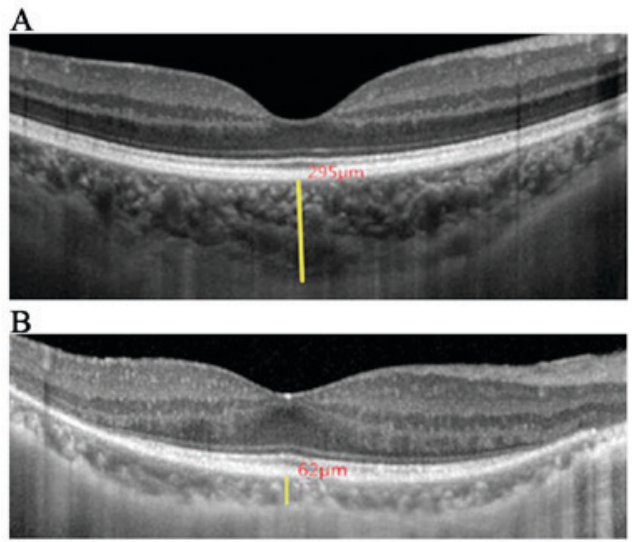

C

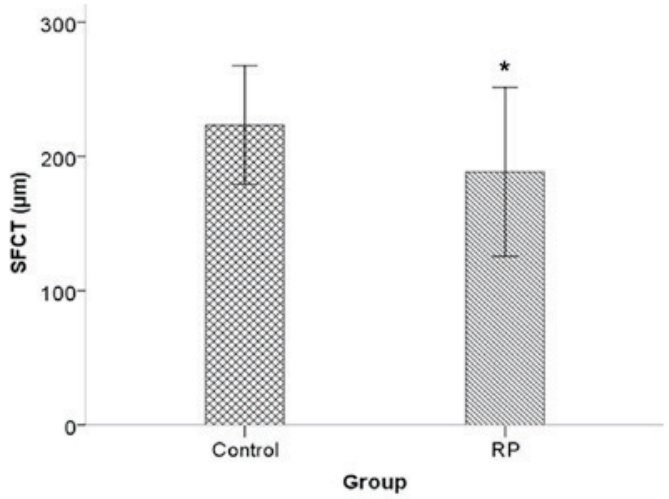

Figure 6. SFCT measurements. A representative optical coherence tomography image of (A) a healthy eye and (B) an eye with RP demonstrate SFCT measurements from the inner border of the sclera to the outer border of the retinal pigment epithelium vertically (yellow line). The mean SFCT was $223.42 \pm 44.16 \mu \mathrm{m}$ in the control group and $193.82 \pm 62.55 \mu \mathrm{m}$ in the RP group. (C) The mean SFCT was significantly thinner in the RP group eyes compared with the control group eyes $(\mathrm{P}<0.05)$. ${ }^{\mathrm{P}}<0.001$ vs. control. SFCT, subfoveal choroidal thickness; $\mathrm{RP}$, retinal pigmentosa.

A

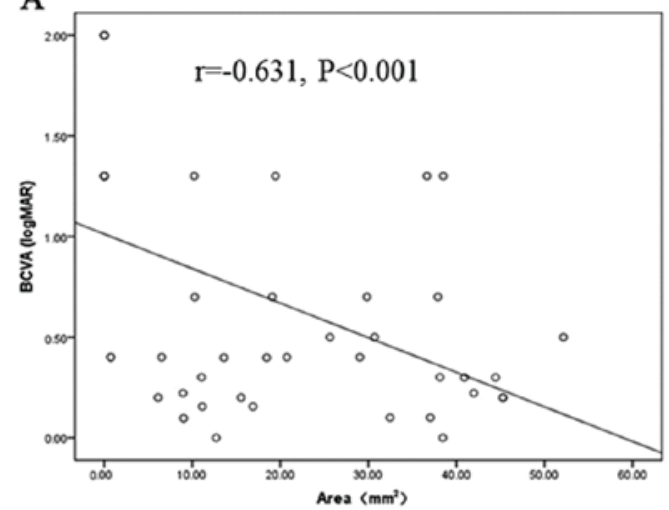

c

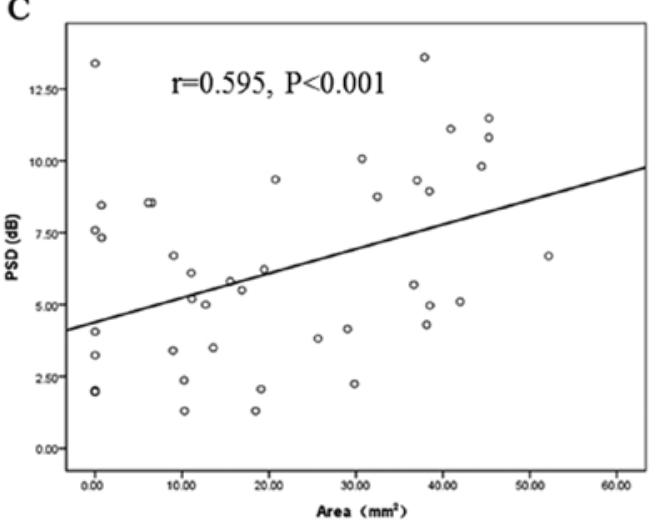

B

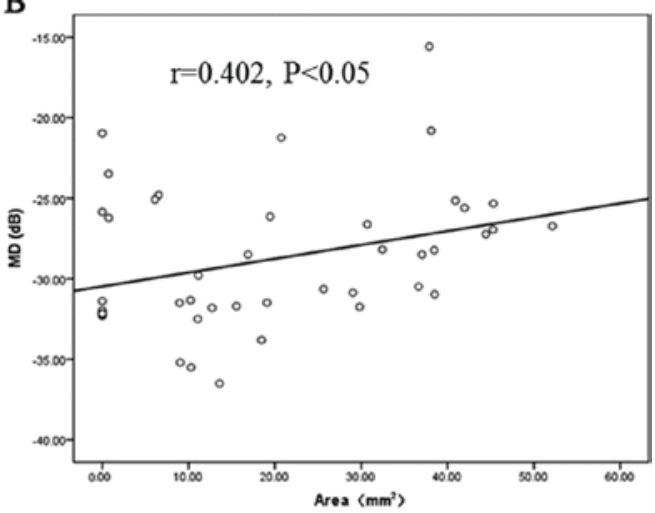

D

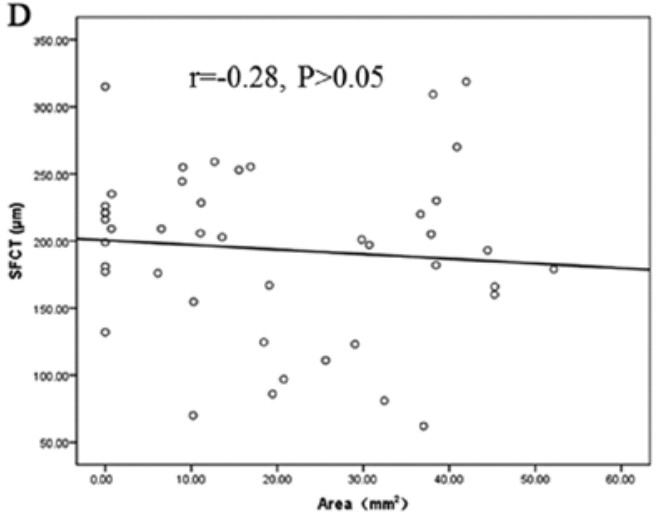

Figure 7. Correlations between the macular-sparing area and the BCVA, visual field and SFCT. (A) There was a significant positive correlation between the macular-sparing area and BCVA ( $\mathrm{r}=-0.631 ; \mathrm{P}<0.001)$, and the visual field in terms of $(\mathrm{B}) \mathrm{MD}(\mathrm{r}=0.402 ; \mathrm{P}<0.05)$ and $(\mathrm{C}) \mathrm{PSD}(\mathrm{r}=0.595 ; \mathrm{P}<0.001)$. (D) No significant correlation was identified between the macular-sparing area and SFCT ( $\mathrm{r}=-0.28$; P>0.05). BCVA, best corrected visual acuity; SFCT, subfoveal choroidal thickness; MD, mean deviation; PSD, pattern standard deviation.

Images representing a typical eye from the control and RP groups are presented in Fig. 6A and B, respectively. The SFCT measurements were obtained from the OCT images using a linear measurement tool. The mean SFCT of the control group was $225.47 \pm 60.52 \mu \mathrm{m}$, while the mean SFCT of the RP group was $193.82 \pm 62.55 \mu \mathrm{m}$ (Fig. 6C). The SFCT was significantly thinner in eyes from the RP group when compared with the control group $(\mathrm{P}<0.001)$.
Correlations between the macular-sparing area size and $B C V A$, visual field and SFCT. The correlations between the macular-sparing area and the BCVA, visual field and SFCT were analyzed using a Spearman's rank correlation test (Fig. 7). There was a significant positive correlation between the size of the macular-sparing area and BCVA $(r=-0.631 ; \mathrm{P}<0.001)$, and the visual field in terms of $\mathrm{MD}(\mathrm{r}=0.402 ; \mathrm{P}<0.05)$ and PSD $(r=0.595 ; \mathrm{P}<0.001)$. However, no significant correlation 
was identified between the macular-sparing area and SFCT $(\mathrm{r}=-0.28 ; \mathrm{P}>0.05)$. These results indicate that the size of the macular-sparing area is an effective parameter by which to assess visual function.

\section{Discussion}

In clinical practice, certain patients with RP are able to maintain a relatively normal macular area for an extended period of time. This normal region has been defined as the 'macular-sparing area' throughout the present study. As patients with RP often have complications, including cataracts (17), ERMs (18) and macular edema (19), it can be difficult to acquire useful details on the fundus from regular fundus photography.

Improvements in technology may lead to advancements within the field of ophthalmic imaging; the present study introduces multicolor imaging as a novel modality that offers improved visualization of macular clinical features in patients with RP. Multicolor imaging utilizes multiple colored lasers to obtain information originating from different retinal structures simultaneously; it is able to reveal topographic changes within different retinal layers. It is difficult to demonstrate these detailed alterations using traditional fundus photography due to refractive stroma turbidity. In the present study, the potential of multicolor imaging as a means for evaluating the clinical features of the macular-sparing area and complications in patients with RP was investigated. To the best of our knowledge, this is the first time Spectralis SD-OCT has been used to observe the characteristics of RP.

In the present study multicolor imaging was used to visualize the macular-sparing area and variable macular changes in patients with RP with various complications, including an ERM and cataracts. Multicolor cSLO imaging demonstrated the radiating retinal folds and traction retinal area despite the presence of cataracts. While the margins of the macular-sparing area were relatively indistinct on traditional fundus photography, the oblong margins of the area were well defined on the multicolor images. Multicolor images also depicted the macular-sparing area with a normal reflective area surrounded by a hyporeflective or hyperreflective ring, which coincided with the outer retinal changes. Multicolor imaging was revealed to possess marked advantages over traditional fundus photography, as it revealed detailed information about retinal structural changes and depicted retinal alterations at a high resolution.

The IR reflectance laser provides detailed images of the choroid, RPE and photoreceptors, which may be used to enhance understanding of retinal degenerative diseases (20). The Spectralis SD-OCT is a novel technology that has been confirmed as an excellent tool for the evaluation of geographic atrophy secondary to age-related macular degeneration (21). The present study identified that the IR reflectance laser image was able to visualize irregular macular alterations, while they were poorly discerned on the blue and green laser reflectance images.

The normal reflective area was identified as being surrounded by either a hyporeflective or hyperreflective ring on the multicolor SLO images. This suggests that certain microstructure anomalous changes may lead to the different appearances of retinal alterations. The present study demonstrated that continuous outer retinal lines were responsible for the normal reflective area. A disrupted ELM line, combined with an invisible EZ line may be presented as a hyporeflective ring surrounding the healthy macular fovea. ELM line thickening as a result of EZ disruption may be associated with the hyperreflective ring, which suggests that ELM remolding occurs to increase the ELM thickness. Outside of the hyporeflective or hyperreflective ring, ELM and EZ lines were absent. A morphologic association between the features on multicolor images and the outer retinal changes on the OCT scans has been identified. Thus, multicolor imaging may be a useful method of detecting subtle changes and providing structural information that could previously only be revealed through an OCT examination.

As the macular-sparing area reflects the outer retinal line changes, it was hypothesized that an association between the macular-sparing area and visual function exists. The results of the present study revealed a significant correlation between the size of macular-sparing area and BCVA. This is consistent with the results of previous studies, where ELM and EZ lines were identified as being significantly correlated with $\operatorname{BCVA}(5,22,23)$. Patients with advanced RP without outer retinal lines exhibit poorer BCVA. However, certain patients with RP who have disorganized segments exhibit abnormal changes in the outer retinal lines as well as the inner retinal layers (8). This influences the BCVA, resulting in a moderate association between the macular-sparing area and BCVAs. The results of the present study demonstrated that multicolor imaging of the macular-sparing area may be another useful parameter by which to evaluate the visual function of patients with RP.

The visual field is associated with OCT parameters (24) and represents another important method of diagnosing RP. The results of the present study demonstrated that the MD and PSD of the visual field were positively correlated with the size of the macular-sparing area; thus indicating that a larger healthy macular area represents a wider visual field. These results are consistent with previous studies, which concluded that the thickness of the photoreceptor outer segments decreased linearly with the loss of visual field $(25,26)$. However, the visual field is influenced by a number of other factors, including RNFL thickness and patient compliance (27). This means that the correlation between the macular-sparing area and the visual field needs to be investigated in a larger number of individuals with RP to confirm the findings of the present study.

$\mathrm{RP}$ is characterized by the degeneration of photoreceptors and RPE cells, in addition to variable choroid atrophy (28). The IR laser was able to penetrate the deepest retinal layers and provide detailed information on choroidal changes. The outer retinal layers are supplied by choroidal capillaries and it was hypothesized that there may be an association between SFCT and the size of the macular-sparing area. However, while SFCT was identified as being reduced in all individuals within the RP group, there was no significant correlation identified between the SFCT and the size of the macular-sparing area. RPE-derived vascular endothelial growth factor is one of the most important factors involved in the maintenance of normal choriocapillaris $(29,30)$, however, the subfoveal choroidal thickness may be maintained for an extended period of time while the macular-sparing area exists. The outer retinal layers 
may be mainly associated with the choriocapillary layer as opposed to the whole choroid thickness (31), so a device that is able to measure choriocapillary thickness is required. Further research to measure additional sites and the choriocapillary layer is required to confirm the association between choroidal thickness and the size of the macular-sparing area.

In conclusion, the results of the present study indicate that multicolor imaging is an effective, novel approach to defining the macular-sparing area and visualizing macular aberrations in patients with RP. Multicolor imaging is able to identify more subtle retinal alterations, and more objective and quantitative information compared with traditional fundus photography. Multicolor imaging is also able to visualize the intact outer retinal structures regardless of complications, including cataracts and ERM. Therefore, multicolor imaging represents a useful tool to investigate macular structural changes and their association with visual function in patients with RP. It was also identified that there is a significant positive correlation between the size of the macular-sparing area and visual field function and BCVA.

\section{Acknowledgements}

The present study was supported by the Shen Kang Hospital Development Center (grant no. SHDC12010207).

\section{References}

1. Mrejen S, Audo I, Bonnel S and Sahel JA: Retinitis pigmentosa and other dystrophies. Dev Ophthalmol 58: 191-201, 2017.

2. Hartong DT, Berson EL and Dryja TP: Retinitis pigmentosa. Lancet 368: 1795-1809, 2006.

3. Berson EL: Long-term visual prognoses in patients with retinitis pigmentosa: The ludwig von sallmann lecture. Exp Eye Res 85: 7-14, 2007.

4. Flynn MF, Fishman GA, Anderson RJ and Roberts DK: Retrospective longitudinal study of visual acuity change in patients with retinitis pigmentosa. Retina 21: 639-646, 2001.

5. Aizawa S, Mitamura Y, Hagiwara A, Sugawara T and Yamamoto S: Changes of fundus autofluorescence, photoreceptor inner and outer segment junction line, and visual function in patients with retinitis pigmentosa. Clin Exp Ophthalmol 38: 597-604, 2010.

6. Drexler W, Sattmann H, Hermann B, Ko TH, Stur M, Unterhuber A, Scholda C, Findl O, Wirtitsch M, Fujimoto JG and Fercher AF: Enhanced visualization of macular pathology with the use of ultrahigh-resolution optical coherence tomography. Arch. Ophthalmol 121: 695-706, 2003.

7. Sun LW, Johnson RD, Langlo CS, Cooper RF, Razeen MM, Russillo MC, Dubra A, Connor TB Jr, Han DP, Pennesi ME, et al: Assessing photoreceptor structure in retinitis pigmentosa and usher syndrome. Invest Ophthalmol Vis Sci 57: 2428-2442, 2016.

8. Liu G, Li H, Liu X, Xu D and Wang F: Structural analysis of retinal photoreceptor ellipsoid zone and postreceptor retinal layer associated with visual acuity in patients with retinitis pigmentosa by ganglion cell analysis combined with OCT imaging. Medicine (Baltimore) 95: e5785, 2016.

9. Fischer MD, Fleischhauer JC, Gillies MC, Sutter FK, Helbig H and Barthelmes D: A new method to monitor visual field defects caused by photoreceptor degeneration by quantitative optical coherence tomography. Invest Ophthalmol Vis Sci 49: 3617-3621, 2008.

10. Chan TCY, Lam SC, Mohamed S and Wong RLM: Survival analysis of visual improvement after cataract surgery in advanced retinitis pigmentosa. Eye (Lond): Aug 4, 2017 (Epub ahead of print). doi: 10.1038/eye.2017.164.

11. Ikeda Y, Yoshida N, Murakami Y, Nakatake S, Notomi S, Hisatomi T, Enaida $\mathrm{H}$ and Ishibashi T: Long-term surgical outcomes of epiretinal membrane in patients with retinitis. Sci Rep 5: 13078, 2015.
12. Pang CE and Freund KB: Ghost maculopathy: An artifact on near-infrared reflectance and multicolor imaging masquerading as chorioretinal pathology. Am J Ophthalmol 158: 171-178, 2014.

13. Wen Y, Klein M, Hood DC and Birch DG: Relationships among multifocal electroretinogram amplitude, visual field sensitivity, and SD-OCT receptor layer thicknesses in patients with retinitis pigmentosa. Invest Ophthalmol Vis Sci 53: 833-840, 2012.

14. Sergott RC: Retinal segmentation using multicolor laser imaging. J Neuroophthalmol 34 (Suppl): S24-S28, 2014.

15. Zeng J, Li J, Liu R, Chen X, Pan J, Tang S and Ding X: Choroidal thickness in both eyes of patients with unilateral idiopathic macular hole. Ophthalmology 119: 2328-2333, 2012.

16. Lemke S, Cockerham GC, Glynn-Milley C, Lin R and Cockerham KP: Automated perimetry and visual dysfunction in blast-related traumatic brain injury. Ophthalmology 123: 415-424, 2015.

17. Andjelic S, Drašlar K, Hvala A and Hawlina M: Anterior lens epithelium in cataract patients with retinitis pigmentosa scanning and transmission electron microscopy study. Acta Ophthalmol 95: e212-e220, 2017.

18. Fujiwara K, Ikeda Y, Murakami Y, Nakatake S, Tachibana T, Yoshida N, Nakao S, Hisatomi T, Yoshida S, Yoshitomi T, et al: Association between aqueous flare and epiretinal membrane in retinitis pigmentosa. Invest Ophthalmol Vis Sci 57: 4282-4286, 2016.

19. Strong S, Liew G and Michaelides M: Retinitis pigmentosa-associated cystoid macular oedema: Pathogenesis and avenues of intervention. Br J Ophthalmol 101: 31-37, 2017.

20. Semoun O, Guigui B, Tick S, Coscas G, Soubrane G and Souied EH: Infrared features of classic choroidal neovascularisation in exudative age-related macular degeneration. $\mathrm{Br} \mathrm{J}$ Ophthalmol 93: 182-185, 2009.

21. Ben Moussa N, Georges A, Capuano V, Merle B, Souied EH and Querques G: MultiColor imaging in the evaluation of geographic atrophy due to age-related macular degeneration. Br J Ophthalmol 99: 842-847, 2015.

22. Yoon CK and Yu HG: The structure-function relationship between macular morphology and visual function analyzed by optical coherence tomography in retinitis pigmentosa. J Ophthalmol 2013: 821460, 2013.

23. Smith TB, Parker M, Steinkamp PN, Weleber RG, Smith N and Wilson DJ; VPA Clinical Trial Study Group; EZ Working Group: Structure-function modeling of optical coherence tomography and standard automated perimetry in the retina of patients with autosomal dominant retinitis pigmentosa. PLoS One 11: e0148022, 2016.

24. Fischer MD, Fleischhauer JC, Gillies MC, Sutter FK, Helbig H and Barthelmes D: A new method to monitor visual field defects caused by photoreceptor degeneration by quantitative optical coherence tomography. Invest Ophthalmol Vis Sci 49: 3617-3621, 2008.

25. Wakabayashi T, Sawa M, Gomi F and Tsujikawa M: Correlation of fundus autofluorescence with photoreceptor morphology and functional changes in eyes with retinitis pigmentosa. Acta Ophthalmol 88: e177-e183, 2010.

26. Wen Y and Birch DG: Outer segment thickness predicts visual field response to QLT091001 in patients with RPE65 or LRAT mutations. Transl Vis Sci Technol 4: 8, 2015.

27. Rao HL, Raveendran S, James V, Dasari S, Palakurthy M, Reddy HB, Pradhan ZS, Rao DA, Puttaiah NK and Devi S: Comparing the performance of compass perimetry with humphrey field analyzer in eyes with glaucoma. J Glaucoma 26: 292-297, 2017.

28. Miyata M, Hata M, Ooto S, Ogino K, Gotoh N, Morooka S, Hasegawa T, Hirashima T, Sugahara M, Kuroda Y, et al: Choroidal and retinal atrophy of bietti crystalline dystrophy patients with CYP4V2 mutations compared to retinitis pigmentosa patients with eys mutations. Retina 37: 1193-1202, 2017.

29. Saint-Geniez M, Kurihara T, Sekiyama E, Maldonado AE and D'Amore PA: An essential role for RPE-derived soluble VEGF in the maintenance of the choriocapillaris. Proc Natl Acad Sci USA 106: 18751-18756, 2009.

30. Ohlmann A, Scholz M, Koch M and Tamm ER: Epithelial-mesenchymal transition of the retinal pigment epithelium causes choriocapillaris atrophy. Histochem Cell Biol 146: 769-780, 2016

31. Adhi M, Regatieri CV, Branchini LA, Zhang JY, Alwassia AA and Duker JS: Analysis of the morphology and vascular layers of the choroid in retinitis pigmentosa using spectral-domain OCT. Ophthalmic Surg Lasers Imaging Retina 44: 252-259, 2013. 